\title{
OPEN Biochemical and structural characterization of quizalofop-resistant wheat acetyl-CoA carboxylase
}

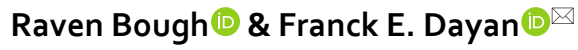

A novel nucleotide mutation in ACC1 resulting in an alanine to valine amino acid substitution in acetyl-CoA carboxylase (ACCase) at position 2004 of the Alopecurus myosuroides reference sequence (A2004V) imparts quizalofop resistance in wheat. Genotypes endowed with the homozygous mutation in one or two ACC1 homoeologs are seven- and 68-fold more resistant to quizalofop than a wildtype winter wheat in greenhouse experiments, respectively. In vitro ACCase activities in soluble protein extracts from these varieties are 3.8- and 39.4-fold more resistant to quizalofop with the homozygous mutation in either one or two genomes, relative to the wildtype. The A2004V mutation does not alter the specific activity of wheat ACCase, suggesting that this resistance trait does not affect the catalytic functions of ACCase. Modeling of wildtype and quizalofop-resistant wheat ACCase demonstrates that the $\mathrm{A} 2004 \mathrm{~V}$ amino acid substitution causes a reduction in the volume of the binding pocket that hinders quizalofop's interaction with ACCase. Docking studies confirm that the mutation reduces the binding affinity of quizalofop. Interestingly, the models suggest that the A2004V mutation does not affect haloxyfop binding. Follow up in vivo and in vitro experiments reveal that the mutation, in fact, imparts negative cross-resistance to haloxyfop, with quizalofop-resistant varieties exhibiting higher sensitivity to haloxyfop than the wildtype winter wheat line.

Acetyl-CoA carboxylase (ACCase or EC 6.4.1.2) catalyzes the first committed and rate-limiting step of fatty acid biosynthesis, converting acetyl-CoA to malonyl-CoA ${ }^{1}$. The three primary functional components of ACCase are biotin carboxylase, biotin carboxyl carrier protein, and carboxyl transferase (see Figure 1 in Takano et al., $2021)^{2}$. Mechanistically, biotin carboxylase utilizes ATP to facilitate the carboxyl group transfer from bicarbonate to the biotin cofactor. The biotin carboxyl carrier protein then moves the newly acquired carboxyl group to the carboxyl transferase domain, where acetyl-CoA is carboxylated to form malonyl-CoA. Most plants have two forms of ACCase, a eukaryotic multifunctional homomeric form located in the cytosol and a prokaryote-like multi-subunit form localized to plastids ${ }^{1}$. Grasses differ from most plants by having only the eukaryotic form of the enzyme in both the cytosol and plastids ${ }^{3,4}$. Group 1 herbicides specifically target the eukaryotic form of ACCase by binding to the carboxyl transferase domain and inhibiting the second catalytic step ${ }^{5}$, whereas these herbicides have little activity on the multi-subunit chloroplastic form. Consequently, group 1 herbicides are excellent graminicides ${ }^{6}$.

The recently commercialized CoAXium ${ }^{\circ}$ winter wheat production system consists of a specialized quizalofop formulation under the tradename Aggressor ${ }^{\circledast}$ for use on wheat varieties with the AXigen ${ }^{\circledast}$ quizalofop resistance trait coupled with a stewardship program aiming to delay resistance in weeds ${ }^{7}$. Quizalofop, applied as the proherbicide form quizalofop-p-ethyl, controls grasses postemergence. Following cuticle penetration, the proherbicide is bioactivated to the active form quizalofop acid in planta. Plants transport the active quizalofop to meristematic tissue, which contains a large concentration of plastid-bound ACCase. Plastid-bound wheat ACCase encoded by the gene ACC1 occurs as a homodimer with three functional domains per monomer ${ }^{8,9}$. Although the binding of quizalofop to ACCase has not been studied previously, work on other aryloxyphenoxy-propionate herbicides demonstrates that these are reversible, noncompetitive inhibitors of grass ACCase ${ }^{10,11}$. All ACCase inhibitors (aryloxyphenoxy-propionates, cyclohexandiones and phenylpyrazolines) interact with the same binding domain on the carboxyl transferase portion of the multifunctional form of ACCase ${ }^{12,13}$. 


\begin{tabular}{|c|c|c|c|c|}
\hline Herbicide ae & Mutant ACC1 Homoeologs & $\mathrm{GR}_{50}\left(\mathrm{~g}\right.$ ae ha $\left.\mathrm{h}^{-1}\right)$ & $R: S^{c}$ & $\mathrm{R}: \mathrm{S} p$-value ${ }^{\mathrm{d}}$ \\
\hline \multirow{3}{*}{ Quizalofop } & 0 & $7.13(1.0)$ & - & - \\
\hline & 1 & $50.1(5.8)$ & $7.0(1.31)$ & $<0.0001$ \\
\hline & 2 & $485(43.6)$ & $68.0(11.6)$ & $<0.0001$ \\
\hline \multirow{3}{*}{ haloxyfop } & 0 & $125(28.7)$ & - & - \\
\hline & 1 & $40.4(13.4)$ & $0.32(0.130)$ & $<0.0001$ \\
\hline & 2 & $26.5(6.1)$ & $0.21(0.07)$ & $<0.0001$ \\
\hline
\end{tabular}

Table 1. Relative herbicide dose effects on whole-plant growth by genotype ${ }^{a}$ for $50 \%$ growth reduction $\left(\mathrm{GR}_{50}\right)^{\mathrm{b}}$. ${ }^{\mathrm{a}}$ Genotype refers to the number of homozygous mutations in $A C C 1$ that result in a A2004V amino acid substitution, with up to three possible on group 2 chromosomes in hexaploid wheat $(2 n=6 x)$. ${ }^{b}$ Leaf fresh weight values $(\mathrm{g})$ normalized to percent untreated controls and transformed with Box-Cox transformation per genotype for each herbicide. Quizalofop dose effect modeled with a 2-parameter log-logistic function and haloxyfop dose effect modeled with a 4-parameter Brain-Cousens function to estimate $\mathrm{GR}_{50}$ values. ${ }^{\mathrm{c}}$ Resistance factor, calculated by dividing the $\mathrm{GR}_{50}$ of a quizalofop-resistant genotype by the susceptible genotype $\mathrm{GR}_{50}$. ${ }^{\mathrm{d}}$ Student's t-test $p$-value $(\alpha=0.05)$ of resistance factor ratios.

The AXigen ${ }^{\oplus}$ trait is a mutant quizalofop-resistant wheat ACCase. Researchers discovered the trait via ethyl methanesulfonate (EMS) mutagenesis and subsequent screening for resistance to quizalofop ${ }^{14}$. The nucleotide substitution in ACC1 imparting quizalofop resistance in CoAXium ${ }^{\star}$ wheat corresponds to position 2004 in the ACCase amino acid sequence from the Alopecurus myosuroides reference sequence ${ }^{14}$. This mutation causes an amino acid substitution of an alanine for a slightly larger valine residue in ACCase (A2004V). Initially, the researchers identified three accessions with heterozygous resistance-conferring mutations for each of three ACC1 gene homoeologs in hexaploid wheat $(6 \mathrm{x}=42)$ on chromosome 2 and later developed a line with a homozygous mutation for each homoeolog. Not all homoeologs contribute equally to quizalofop resistance, with the A to $\mathrm{V}$ mutation on the $\mathrm{D}$ homoeolog providing the greatest level of resistance, followed by the same mutation in the $\mathrm{A}$ and $\mathrm{B}$ homoeologs. The Wheat Breeding and Genetics Program at Colorado State University introgressed the mutations from the A and D ACC1 homoeologs into an elite genetic background and developed the initial CoAXium $^{\bullet}$ varieties featuring the mutation in both homoeologs.

Our objectives were to determine if the amino acid substitution affects the specific activity of wheat ACCase and to describe how the substitution imparts resistance at biochemical and structural levels. We further quantified fold-differences in whole-plant and enzymatic resistance between wheat with wildtype ACCase and wheat with one or two mutant ACCase homoeologs.

We first determined whole-plant quizalofop resistance in wheat with one or two mutant ACCase homoeologs by comparison to wildtype, susceptible wheat and correlated these experiments to the effect of quizalofop in in vitro assays of wheat ACCase. We then modeled the changes in the ACCase protein structure and its interaction with the herbicide using homology modelling, molecular dynamic simulations, and docking.

\section{Results}

Herbicide dose effect on plant growth. Three wheat genotypes were grown in the greenhouse to assess the contribution of a homozygous ACC1 mutation in either one or two genomes leading to whole-plant quizalofop resistance relative to the wildtype line, and to examine cross-resistance to haloxyfop. In general, whole-plant resistance to either quizalofop or haloxyfop varies by the number of mutant ACC1 homoeologs present in a wheat genotype. The residual standard error of the 2-parameter log-logistic regression model with Box-Cox transformation of quizalofop doses is 11.5 with 97 degrees of freedom. For the haloxyfop doses, the residual standard error of the 4-parameter Brain-Cousens model with Box-Cox transformation is 2.14 with 92 degrees of freedom.

The estimated quizalofop doses that reduce fresh biomass growth normalized as a percent of untreated controls by $50 \%\left(\mathrm{GR}_{50}\right)$ range from 7.13 to $485 \mathrm{~g}$ ae ha $^{-1}$, while haloxyfop $\mathrm{GR}_{50}$ values range from 26.5 to $125 \mathrm{~g}$ ae ha $\mathrm{h}^{-1}$ (Table 1 and Fig. 1). Based on $\mathrm{GR}_{50}$ values, genotypes with the homozygous mutation in either one or two genomes are 7.0 and 68.0 times more resistant to quizalofop than the wildtype, susceptible genotype, respectively. The quizalofop-resistant genotypes are more sensitive to haloxyfop than the wildtype. Lines with the mutation in either one or two of the genomes are about three-fold and five-fold more sensitive to haloxyfop, respectively, relative to the susceptible genotype (Table 1). According to Student's t-tests, these genotypes are significantly different from the susceptible genotype for both herbicides.

Enzyme specific activity assay. Crude enzyme preparations containing ACCase activity, the target enzyme of quizalofop and other group 1 herbicides, were extracted from wheat to compare specific activity and to determine enzyme-level resistance of the same three genotypes used in the whole-plant resistance studies. Enzyme extract specific activity range from 2.66 to $3.91 \mathrm{nmol} \mathrm{mg} \mathrm{protein}{ }^{-1}$ (Fig. 2). Values are not significantly different between genotypes $\left(\mathrm{F}_{2,6}=0.278\right)$.

Herbicide dose effect on ACCase activity. Enzyme-level resistance to either quizalofop or haloxyfop varies by the number of mutant ACC1 homoeologs present in a wheat genotype. The residual standard error of 

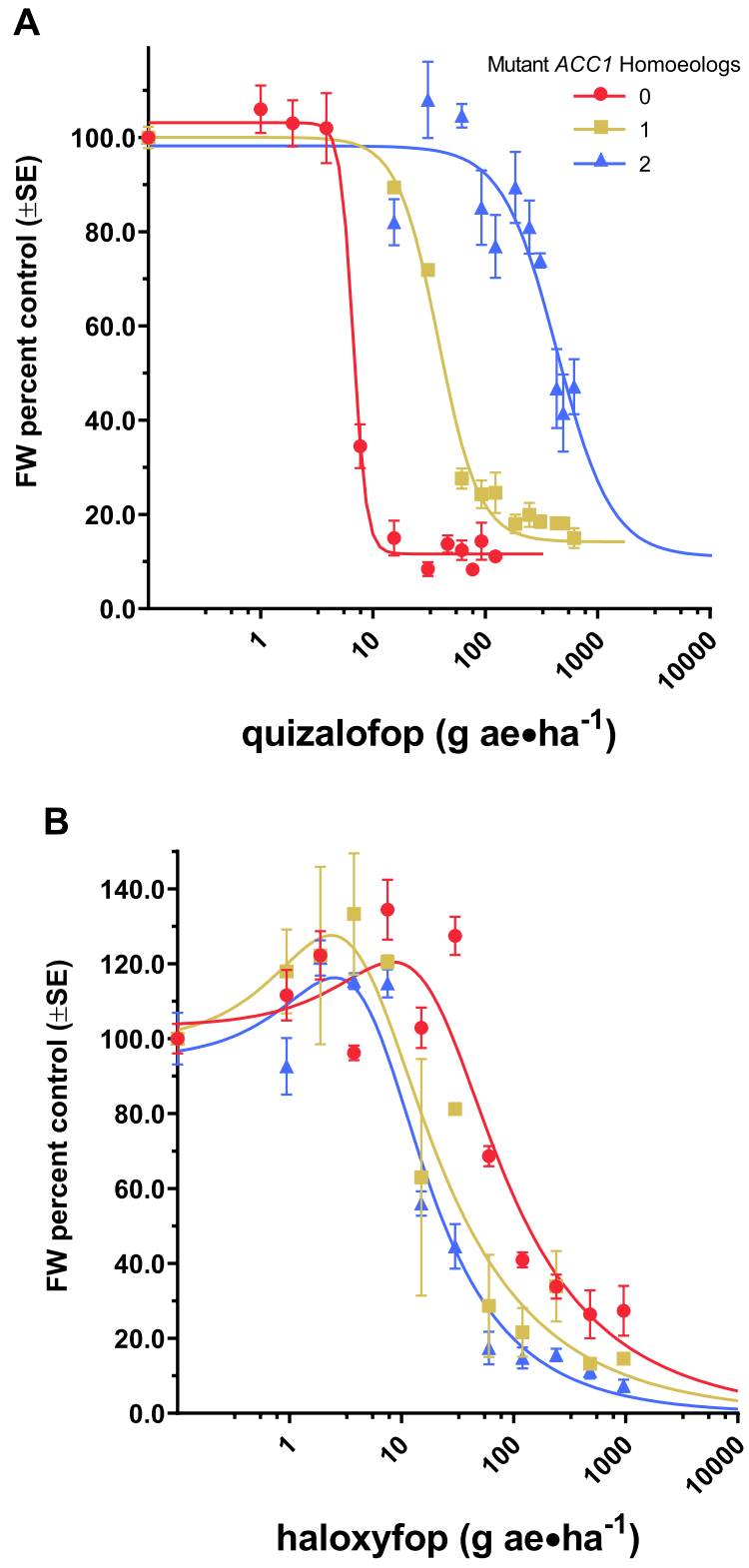

Figure 1. Models $\mathrm{s}^{\mathrm{a}}$ of quizalofop (A) and haloxyfop (B) herbicide dose effects on whole-plant growth by genotype ${ }^{\mathrm{b}}$. ${ }^{\mathrm{a}}$ Leaf fresh weight values $(\mathrm{g})$ normalized to percent untreated controls and transformed with Box-Cox transformation per genotype for each herbicide. Quizalofop dose effect modeled with a 2-parameter log-logistic function (A) and haloxyfop dose effect modeled with a 4-parameter Brain-Cousens function

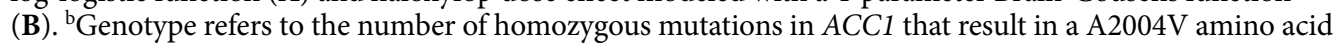
substitution, with up to three possible on group 2 chromosomes in hexaploid wheat $(2 n=6 x)$. These mutations result in quizalofop-resistant wheat ACCase.

the 2-parameter log-logistic regression model with Box-Cox transformation of quizalofop doses is 0.435 with 48 degrees of freedom. A similar model of haloxyfop doses has a residual error of 0.940 with 55 degrees of freedom.

The estimated quizalofop doses that inhibit ACCase activity normalized as a percent of untreated controls to $50 \%\left(\mathrm{I}_{50}\right)$ range 0.486 to $19.3 \mu \mathrm{M}$, while haloxyfop $\mathrm{I}_{50}$ values range from 0.968 to $7.63 \mu \mathrm{M}$ (Table 2 and Fig. 3 ). ACCase extracts from invididuals with the resistance mutation in one or two genomes are 3.8 and 39.4 times more resistant to quizalofop than the wildtype, susceptible genotype, respectively, based upon estimated $\mathrm{I}_{50}$ values. Conversely, ACCase extracts from the same quizalofop-resistant genotypes are less resistant to haloxyfop than the susceptible genotype. These genotypes are between seven- and eight-fold more sensitive to haloxyfop than the wildtype (Table 2 and Fig. 3).

While the resistance factor for lines with the AXigen trait in two genomes is statistically significant for quizalofop in Student's t-test, the resistance factors for lines with a single resistant homoeolog is not statistically 


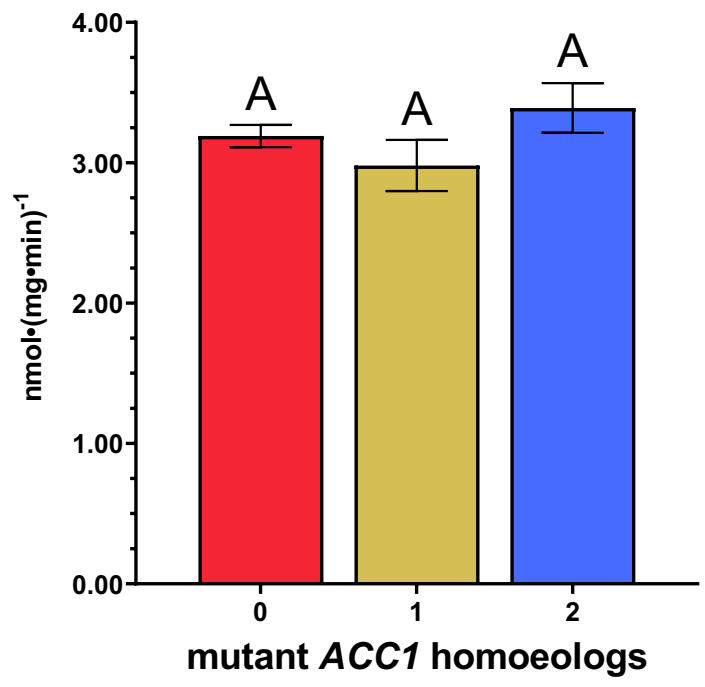

Figure 2. ACCase specific activity by genotype ${ }^{\mathrm{a}, \mathrm{b}}$. ${ }^{\mathrm{a}} \mathrm{Genotype}$ refers to the number of homozygous mutations in ACC1 that result in a A2004V amino acid substitution, with up to three possible on group 2 chromosomes in hexaploid wheat $(2 \mathrm{n}=6 \mathrm{x})$. These mutations result in quizalofop-resistant wheat ACCase. ${ }^{\mathrm{b}}$ Genotype specific activities labeled with the same capital letter are not significantly different according to Student's t-tests $(\alpha=0.05)$.

\begin{tabular}{|c|c|c|c|c|}
\hline Herbicide ae & Mutant $A C C 1$ Homoeologs & $\mathbf{I}_{50}(\mu \mathrm{M})$ & $R: S^{c}$ & $R: S p$-value ${ }^{d}$ \\
\hline \multirow{3}{*}{ Quizalofop } & 0 & $0.49(0.13)$ & - & - \\
\hline & 1 & $1.84(0.52)$ & $3.8(1.5)$ & 0.0634 \\
\hline & 2 & $19.30(4.01)$ & $39.4(13.3)$ & 0.00571 \\
\hline \multirow{3}{*}{ Haloxyfop } & 0 & $7.63(1.08)$ & - & - \\
\hline & 1 & $0.97(0.21)$ & $0.13(0.003)$ & $<0.0001$ \\
\hline & 2 & $1.12(0.25)$ & $0.15(0.004)$ & $<0.0001$ \\
\hline
\end{tabular}

Table 2. Relative herbicide dose effects on ACCase activity by genotype ${ }^{\mathrm{a}}$ for $50 \%$ inhibition $\left(\mathrm{I}_{50}\right)^{\mathrm{b}}$. ${ }^{\mathrm{a}} \mathrm{Genotype}$ refers to the number of homozygous mutations in ACC1 that result in a A2004V amino acid substitution, with up to three possible on group 2 chromosomes in hexaploid wheat $(2 n=6 x)$. These mutations result in quizalofop-resistant wheat ACCase. ${ }^{\mathrm{b}}$ Activity values (DPM) normalized to percent untreated controls and transformed with Box-Cox transformation per genotype for each herbicide. The dose effect of each herbicide is modeled with a 2-parameter log-logistic function to estimate $I_{50}$ values. ${ }^{c}$ Resistance factor, calculated by dividing the $\mathrm{I}_{50}$ of a quizalofop-resistant genotype by the susceptible genotype $\mathrm{I}_{50}$. ${ }^{\mathrm{d}}$ Student's t-test $p$-value $(\alpha=0.05)$ of resistance factor ratios.

significant although the $p$-value is close to the significance threshold. Resistance to haloxyfop in these genotypes is significantly different from the susceptible genotype.

The resistant factors at the enzyme level are approximately half of those measured on whole plant dose-response curves (Tables 1 and 2). The higher level of resistance in planta, relative to the in vitro activity, is common because plants have additional mechanisms to cope with the presence of herbicides, such as reduced foliar absorption and metabolic degradation of xenobiotics ${ }^{15}$.

Nonetheless, our results suggest that quizalofop resistance conferred by the AXigen ${ }^{\otimes}$ trait is additive at both the whole-plant and enzyme-level. Estimated $\mathrm{GR}_{50}$ values in the whole-plant dose response trials for the susceptible and single-mutant genotype were within confidence intervals provided by Ostlie et al. in $2015^{14}$, however, our resistance factor estimate for the single-mutant genotype based on these values is nearly double.

Wheat lines with the quizalofop-resistance trait are more sensitive to haloxyfop relative to the susceptible genotype in both whole-plant and enzyme-level dose-response trials. These results suggest that while the ACCase conformational change induced by the A2004V amino acid substitution increases quizalofop-resistance, the change causes negative cross-resistance to haloxyfop (e.g., increases susceptibility to haloxyfop). The negative cross-resistance to haloxyfop is not concerning for the CoAXium ${ }^{\circledR}$ Wheat Production System because the system is only available in the United States, where haloxyfop is not labeled for use in wheat.

Homology modeling of wheat carboxyl transferase domain and docking of quizalofop and haloxyfop. Both quizalofop and haloxyfop are group 1 herbicides that target multifunctional eukaryotic forms of ACCase. However, our biological studies in the greenhouse and biochemical characterization of 

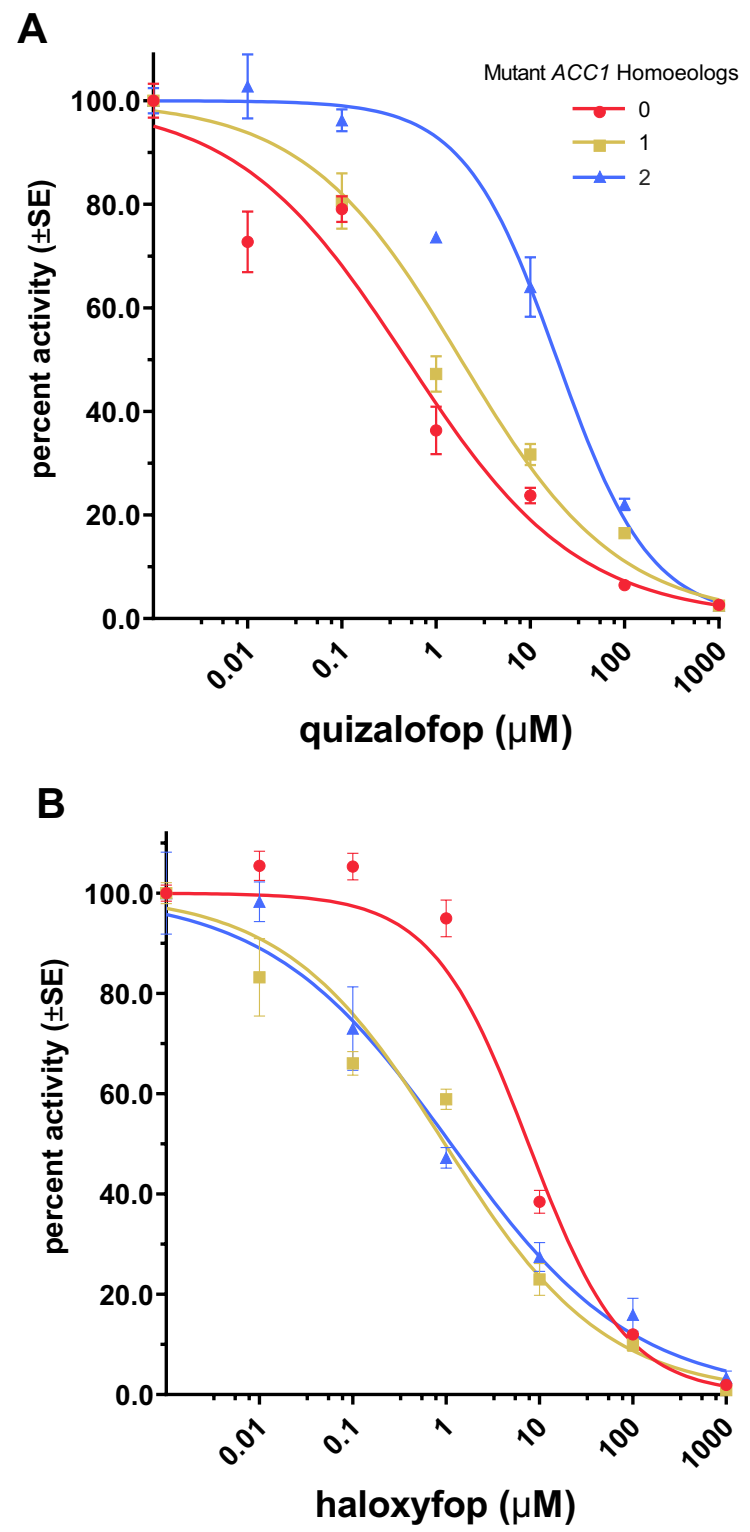

Figure 3. Models ${ }^{\mathrm{a}}$ of quizalofop (A) and haloxyfop (B) herbicide dose effects on ACCase activity by genotype . $^{\mathrm{b}}$. ${ }^{\mathrm{a}}$ Genotype refers to the number of homozygous mutations in ACC1 that result in a A2004V amino acid substitution, with up to three possible on group 2 chromosomes in hexaploid wheat $(2 n=6 x)$. These mutations result in quizalofop-resistant wheat ACCase. ${ }^{\mathrm{b}}$ Activity values (DPM) normalized to percent untreated controls and transformed with Box-Cox transformation per genotype for each herbicide. The dose effect of each herbicide is modeled with a 2-parameter log-logistic function.

ACCase reveal that the alanine to valine mutation imparts tolerance to quizalofop both in planta and in vivo, while causing negative cross-resistance to haloxyfop.

Although quizalofop and haloxyfop are structurally related (Fig. 4A,B), quizalofop is a slightly larger molecule due to its heterobicyclic 6-chloro-quinoxalin-2-yl rings relative to the monocyclic 3-chloro-5-(trifluoromethyl) pyridin-2-yl ring of haloxyfop, with CPK volumes of 303.97 and $317.09 \mathrm{~A}^{3}$, respectively (Table 3). Quizalofop has a smaller molecular weight than haloxyfop despite a larger volume. Both herbicides are lipophilic, although quizalofop is more so according to a greater partition coefficient $(\log P)$. Quizalofop has a slightly larger dipole moment than haloxyfop, indicating it is more polar. The herbicide molecules have a similar electrostatic potential (Fig. 4C,D).

The calculated binding energy of quizalofop increases from -6.07 to $-5.70 \mathrm{kcal} \mathrm{mol}^{-1}$, whereas the calculated binding energy of haloxyfop decreases slightly from -5.074 to $-5.211 \mathrm{kcal} \mathrm{mol}^{-1}$ (Table 4 ). The number of correct docking poses and root-mean-square deviation of atomic poses for all docking combinations are within acceptable limits. Clearly, both quizalofop and haloxyfop bind to the catalytic domain of the wildtype carboxyl transferase (Fig. 5A,C). The A2004V amino acid substitution is located at the bottom left of the herbicide binding domain, where the methyl side chain of alanine occupies a smaller volume than that of the isopropyl side chain of 

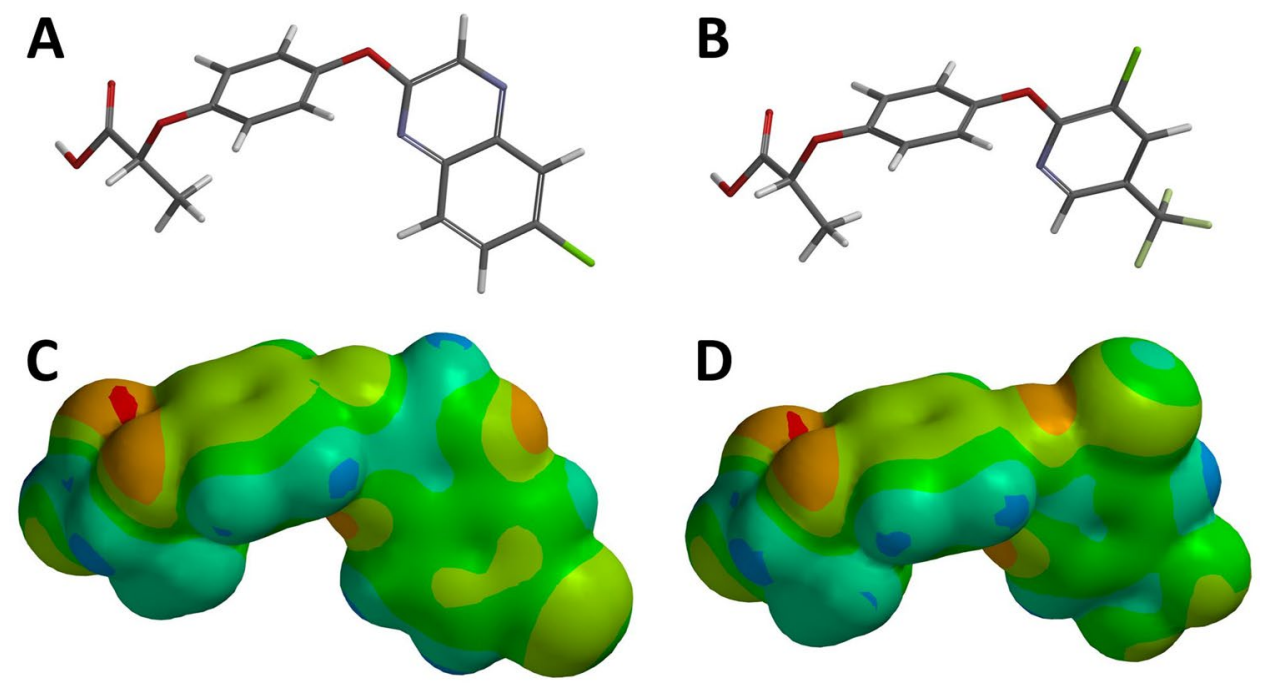

Figure 4. Structures of (A) quizalofop and (B) haloxyfop showing the carbons in gray, hydrogen in white, oxygen in red, nitrogen in blue, chlorine in green and fluorine in cyan on the left side. Electrostatic potential maps of (C) quizalofop and (D) haloxyfop. Colors on the volume represent range from electronegative (red) to electropositive (blue) partial charges. Images were generated using Spartan'20 version 1.1.2 (Wavefunction, Inc., Irvine, CA 92612) (https://www.wavefun.com/).

\begin{tabular}{|l|c|c|}
\hline Parameters & Quizalofop & Haloxyfop \\
\hline $\mathrm{Mw}$ & 344.75 & 361.70 \\
\hline $\log P$ & 1.89 & 0.65 \\
\hline CPK volume A & 317.09 & 303.97 \\
\hline Energy $\left(1000 \mathrm{kj} \mathrm{mol}^{-1}\right)$ & -4006.2 & -4445.5 \\
\hline Dipole moment & 3.51 & 3.08 \\
\hline
\end{tabular}

Table 3. Physicochemical properties of quizalofop and haloxyfop.

\begin{tabular}{|c|c|c|}
\hline & Quizalofop & Haloxyfop \\
\hline & \multicolumn{2}{|c|}{ Wildtype wheat ACCase } \\
\hline Correct docking poses & $92 / 110$ & $82 / 110$ \\
\hline Calculated binding energy $^{\mathrm{a}}$ & $-6.070 \pm 0.095$ & $-5.074 \pm 0.206$ \\
\hline \multirow[t]{2}{*}{ RMSD $^{\mathrm{b}}$} & $0.273 \pm 0.021$ & $0.245 \pm 0.033$ \\
\hline & \multicolumn{2}{|c|}{$\begin{array}{l}\text { Quizalofop-resistant wheat } \\
\text { ACCase }\end{array}$} \\
\hline Correct docking pose & $95 / 110$ & $104 / 110$ \\
\hline Calculated binding energy & $-5.700 \pm 0.230$ & $-5.211 \pm 0.221$ \\
\hline RMSD & $0.434 \pm 0.034$ & $0.242 \pm 0.035$ \\
\hline
\end{tabular}

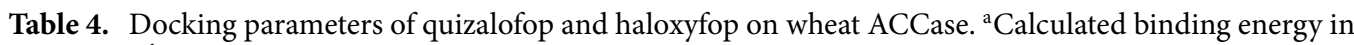
$\mathrm{kcal} \mathrm{mol}^{-1}$. ${ }^{\mathrm{b}} \mathrm{RMSD}$ relative to initial coordinate of quizalofop or haloxyfop based on the crystal structure of 1UYS.

valine. The asterisk in Fig. 5B highlights the strong steric hindrance between the volume occupied by the valine side chain and the 6-chloro-quinoxalin-2-yl group of quizalofop. The same A2004V amino acid substitution does not prevent binding of haloxyfop (Fig. 5D).

\section{Discussion}

Group 1 herbicides are reversible, noncompetitive inhibitors of the multifunctional form of ACCase ${ }^{10,11}$. Since grass plants only possess this form, group 1 herbicides are excellent graminicides ${ }^{16,17}$. Whole-plant quizalofop dose greenhouse experiments confirm resistance in quizalofop-resistant wheat genotypes (Table 1). 


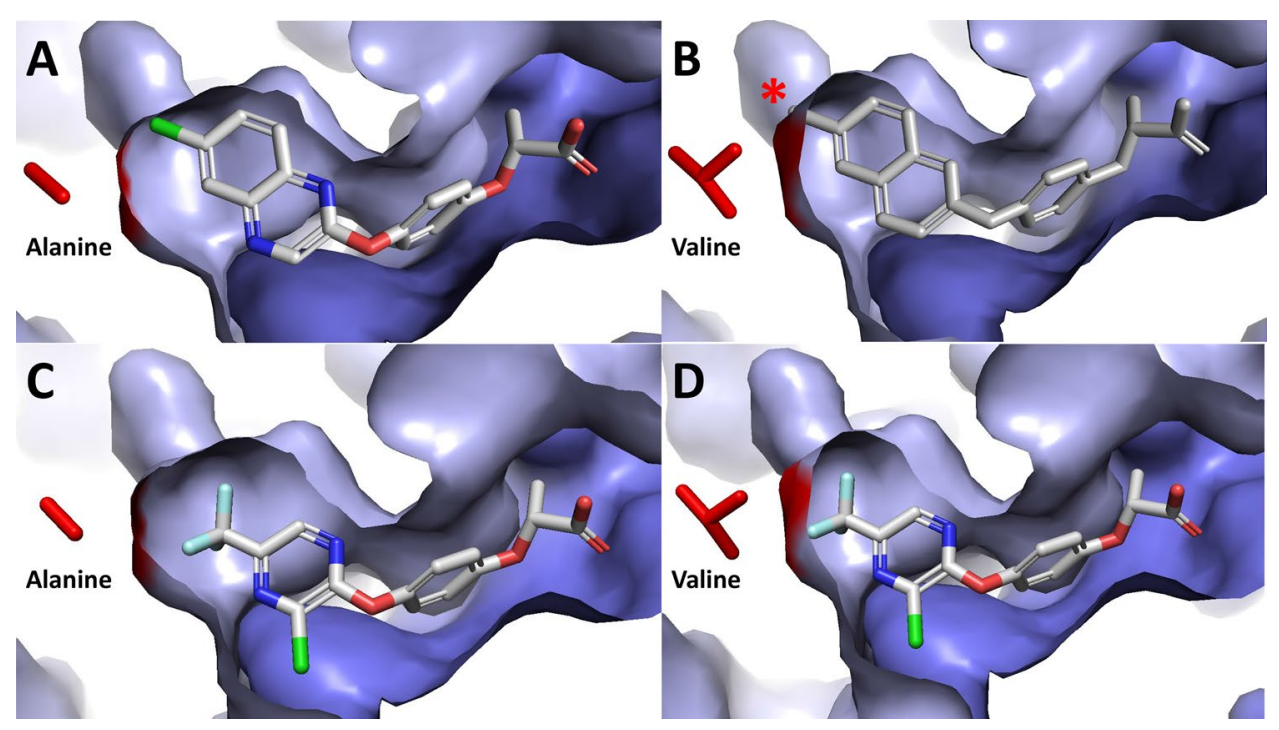

Figure 5. Conformational change caused by the alanine to valine amino acid substitution at position 2004 affects the binding of quizalofop to the carboxyl transferase domain of ACCase. Docking of quizalofop and haloxyfop in the wild-type wheat carboxyl transferase domain are shown in panels (A,C) with the methyl group of the alanine side chain shown in red. Changes to the binding cavity caused by the valine substitution (isopropyl group in red) are shown in panels (B,D). The small reduction in the volume of the binding domain introduces strong steric hinderance (as denoted by asterisk) that prevents quizalofop from binding (panel C). The structure of quizalofop is shown in white solely illustrate the steric hindrance because the herbicide no longer binds in this domain. On the other hand, the valine mutation does not interfere with haloxyfop binding (panel D). Images were generated using PyMOL version 2.3.3 (Schrödinger, New York, NY 10036) (https://www. schrodinger.com/products/pymol).

Dose-response curves shift right with an increasing number of ACC1 resistance alleles (Fig. 1A), therefore having the mutation in two genomes is more resistant than if present in a single genome, and the level of quizalofop resistance imparted by the AXigen ${ }^{\oplus}$ trait appears to be additive. The level of resistance to quizalofop at the enzyme-level parallels the plant responses in the greenhouse study (Table 2 and Fig. 3A), establishing a strong linear relationship between the number of mutant resistant homoeologs with the phenotypic and enzymatic responses.

The lack of significant differences in specific activity between genotypes with susceptible $A C C 1$ and one or two resistant ACC1 homoeologs suggests that the conformational change induced by the A2004V amino acid substitution does not affect ACCase activity. This supports field trials data reporting comparable CoAXium ${ }^{\circ}$ wheat yields following quizalofop treatment to non-treated, quizalofop-susceptible wheat yields (data not shown).

Several structures of the multifunctional form of ACCase have been co-crystalized with inhibitors from the three main families of herbicides from this group (i.e., aryloxyphenoxy-propionates, cyclohexandiones and phenylpyrazolines). Structures reveal that these inhibitors hold a similar pose in the binding domain on the carboxyl transferase portion of the multifunctional form of ACCase ${ }^{12,13}$. The location of the A2004V mutation imparting resistance to quizalofop in wheat is within the same binding domain targeted by ACCase inhibitors ${ }^{12,13}$. While an amino acid substitution from alanine to valine does not affect the overall architecture of the carboxyl transferase domain, it imparts a local conformational change that reduces the volume of the binding pocket. This relatively small change impacts the binding of quizalofop because of its large heterobicyclic 6-chloroquinoxaline group (Fig. 5A,B) whereas it does not affect the binding of haloxyfop with its smaller monocyclic 3-chloro-5(trifluoromethyl)pyridin-2-yl group (Fig. 5C,D). This was further supported by computational binding energy calculation. The mutation increased the calculated binding energy of quizalofop, relative to the wildtype, whereas this same mutation lowered the calculated binding energy of haloxyfop. Follow-up experiments confirmed these computational observations, where quizalofop-resistant genotypes are more sensitive to haloxyfop than the wildtype genotype in both whole-plant (Table 1) and enzyme-level (Table 2) dose-response studies. The leftward shift of whole-plant (Fig. 1B) and enzyme activity (Fig. 3B) dose-response curves with an increasing number of resistance mutations indicates that the quizalofop resistance mutation imparts negative cross-resistance to haloxyfop. The negative cross-resistance to haloxyfop is not concerning for the CoAXium ${ }^{\infty}$ Wheat Production System because the system is only available in the United States, where haloxyfop is not labeled for use in wheat.

In conclusion, the CoAXium wheat production system is a new tool for management of difficult to control winter weeds $s^{7,18}$. The AXigen ${ }^{\oplus}$ resistance trait is based on an alanine to valine mutation at position 2004 of ACCase which hinders the binding of quizalofop. An unexpected outcome of this research is the discovery that the AXigen trait imparts negative cross-resistance to haloxyfop. Further studies could identify other group 1 inhibitors that may be developed as tools to efficiently control volunteer CoAXium wheat. 


\section{Methods}

Plant material and growing conditions. Four wheat genotypes were used to compare whole-plant resistance of lines with wildtype and mutant ACCase as well as specific activity and resistance of extracted ACCase. The AF10 and Hatcher lines were provided by Dr. Scott Haley from the Department of Crop and Soil Science, and the varieties Byrd, and Incline AX were obtained from the Colorado Wheat Research Foundation. All experiments carried out on these wheat varieties were done with their permission and complied with local and national regulations. Byrd is a quizalofop-susceptible variety that was the recurrent parent for Incline AX, a CoAXium ${ }^{ø}$ variety, whereas Hatcher is the original mutagenized variety. AF10 is one of the initial quizalofopresistant lines, which contains a homozygous mutant ACC1 homoeolog in sub-genome $\mathrm{D}^{10}$. Incline AX exhibits two mutant homoeologs in sub-genomes A and D. Byrd was the wildtype, null mutant in all physical experiments except for the quizalofop plant growth dose-response experiment, where Hatcher was substituted. AF10 and Incline AX were consistently used as representative lines with the quizalofop-resistance trait in one and two genomes, respectively, in the same experiments. Plants for the whole-plant dose responses and ACCase extractions were grown from seed in soilless media. Plants were maintained in a greenhouse environment with temperatures from 20 to $25^{\circ} \mathrm{C}$, relative humidity between 50 and $70 \%$, and a 14-h daylength with natural lighting supplemented by sodium halide lamps.

Herbicide dose effect on plant growth. Prior to reaching the jointing stage, wheat seedlings were treated with herbicide. Untreated seedlings of each line were also retained to normalize data. Eleven rates of herbicide were used in each dose-response experiment. Quizalofop herbicide doses for the susceptible line ranged from 3.85 to $108 \mathrm{~g}_{\mathrm{ae} \mathrm{ha}} \mathrm{ha}^{-1}$, whereas doses for resistant lines ranged from 15.4 to $493 \mathrm{~g} \mathrm{ae} \mathrm{ha}^{-1}$. Haloxyfop doses ranged from 15.4 to $616 \mathrm{~g}$ ae ha ${ }^{-1}$ for all wheat lines. Non-ionic surfactant was added to each dose $(0.25 \% \mathrm{v} / \mathrm{v})$. All treatments were applied in a single pass at $40 \mathrm{~cm}$ above the plant canopy using a spray chamber. Each treatment was triplicated, with four plants comprising a replicate. Fresh leaf biomass was harvested three weeks after treatment. Biomass was adjusted in some instances to account for germination of less than four plants per replicate.

Enzyme extraction. Approximately $5.0 \mathrm{~g}$ of crown tissue was harvested from each genotype at the two-leaf stage, flash frozen in liquid nitrogen, and stored at $-80^{\circ} \mathrm{C}$. Enzyme extraction and activity assay were modified from previously published methods ${ }^{19-21}$. Frozen wheat tissue was powdered in liquid nitrogen. All subsequent extraction steps were carried out on ice or in a cold room to maintain an extract temperature of $4{ }^{\circ} \mathrm{C}$. Ground tissue was transferred to $14.85 \mathrm{~mL}$ extraction buffer (100 mM Trizma, $20 \mathrm{mM} \mathrm{DTT}, 2 \mathrm{mM}$-ascorbic acid, $1 \mathrm{mM}$ EDTA, 0.5\% w/v PVP-40, 0.5\% w/v insoluble PVP-20, and 10\% v/v glycerol; pH 8.0) and $150 \mu \mathrm{L}$ of $100 \mathrm{mM}$ PMSF (dissolved in $100 \%$ ethanol). The tissue was homogenized at max speed for $30 \mathrm{~s}$ with a homogenizer probe. Homogenate was filtered by hand with Miracloth, followed by centrifugation for $30 \mathrm{~min}$ at a speed of $25,000 \times \mathrm{g}$. The resulting supernatant was slowly brought to $66 \%$ saturation with solid ammonium sulfate and stirred for an hour.

Ammonium sulfate solutions were centrifuged using the same parameters as the previous centrifugation step to precipitate ACCase and similar proteins. After discarding the supernatant, the protein pellets were resuspended with $2.5 \mathrm{~mL}$ elution buffer $\left(50 \mathrm{mM}\right.$ tricine, $50 \mathrm{mM} \mathrm{KCl}, 2.5 \mathrm{mM} \mathrm{MgCl}_{2}$, and $\left.1 \mathrm{mM} \mathrm{DTT}^{\mathrm{mH}}=8.0\right)$. The protein extracts were desalted using a gravity protocol with conditioned PD-10 columns. Desalted protein extracts were eluted with $3.5 \mathrm{~mL}$ of elution buffer and mixed with $25 \% \mathrm{v} / \mathrm{v}$ glycerol.

Enzyme specific activity assay. To determine ACCase specific activity, four $40 \mu \mathrm{L}$ aliquots of fresh protein extract per genotype were preincubated for $3 \mathrm{~min}$ in assay solution without acetyl-CoA. Acetyl-CoA was subsequently added to three aliquots to initiate enzyme activity, whereas water was added in lieu of acetyl-CoA for a background readings. Preincubation and reaction steps were maintained at $32{ }^{\circ} \mathrm{C}$ with moderate shaking. Final concentrations of assay solution components for each $200 \mu \mathrm{L}$ reaction (including enzyme aliquot) were as follows: $20 \mathrm{mM}$ tricine ( $\mathrm{pH}=8.3$ ), $10 \mathrm{mM} \mathrm{KCl}, 10 \mathrm{mM} \mathrm{MgCl}_{2}, 5 \mathrm{mM} \mathrm{ATP}, 3.24 \mathrm{mM} \mathrm{NaHCO}$, $2.5 \mathrm{mM}$ DTT, $0.1 \% \mathrm{w} / \mathrm{v} \mathrm{BSA}, 0.25 \mathrm{mM}$ acetyl-CoA, and $0.25 \mathrm{mM} \mathrm{NaH}^{14} \mathrm{CO}_{3}$ (provides $18.5 \mathrm{kBq}$ per reaction). After $10 \mathrm{~min}$, enzyme reactions were quenched with $20 \mu \mathrm{L}$ of $12 \mathrm{M}$ hydrochloric acid. Solutions were transferred to $20 \mathrm{~mL}$ scintillation vials and left uncapped overnight to enable volatilization of unincorporated carbonate to $\mathrm{CO}_{2}$. Reactions were carried out in a fume hood equipped with a ${ }^{14} \mathrm{C}$ filter. The following day, $10 \mathrm{~mL}$ of scintillation cocktail was added to each vial. Vials were capped and vortexed for $20 \mathrm{~s}$ prior to analysis. Radioactivity was measured with a liquid scintillation analyzer in disintegrations per min (DPM).

Herbicide dose effect on ACCase activity. Specific activity dose-responses were conducted using the same protocol as the specific activity assay but with the addition of herbicide immediately preceding the preincubation step. Dilution series were prepared with quizalofop acid and haloxyfop acid analytical standards dissolved in $100 \%$ acetonitrile. Triplicated dose treatments per genotype included background readings (water added in lieu of acetyl-CoA), null herbicide doses, and doses that provided 0.0100, 0.100,1.00,10.0, 100, and $1000 \mu \mathrm{M}$ herbicide in the final assay solution.

Homology modeling of wheat carboxyl transferase domain. The segments of wheat ACCase sequence where the A2004V substitution is located were aligned to the sequence of the CT domain of yeast ACCase using EMBOSS Needle ${ }^{22}$. This information was used to build a homology model of wheat ACCase based on the crystal coordinates of the carboxyl transferase (CT) domain of yeast ACCase (1UYS) ${ }^{12}$ available from the RCSB protein data bank. The homology modeling pipeline was similar to that reported previously 
to model the binding of glufosinate on resistant glutamine synthetase from Lolium perenne ${ }^{23}$. A preliminary model was obtained using Modeller $10.0^{24-26}$. The model was assembled in its functional dimer configuration and refined using GROMACS (version 2018.3) 27,28 $^{27}$ a workstation with two processors (96 threads) and video cards (10 GB GPU memory each). Steric clashes or inappropriate geometries were corrected through molecular dynamics simulation and evaluated using MolProbity ${ }^{29,30}$ as described before.

Proteins and ligand interactions were visualized using PyMOL v.2.3.3. $3^{31}$. Additionally, the binding of quizalofop and haloxyfop to native and mutant carboxyl transferase dimers of wheat was measured using established protocols developed for Autodock ${ }^{32,33}$. Briefly, the receptor was defined to encompass residues lining the herbicide binding domain. Amino acid charges were calculated using Gasteiger charges including all polar hydrogens ${ }^{34-36}$. The docking procedure imposed a covalent map to involve the nitrogen hydrogen of ILE 222 in the docking of the herbicides within the gridbox. A total of 100 poses were generated.

Statistical analysis. All statistical analysis was conducted with packages in RStudio v.1.2.5033 with $\mathrm{R}$ "Orange Blossom"28. To model the effect of herbicide on plant growth, fresh weight data was first normalized to percent of control means. Data was transformed with a Box-Cox transformation. For quizalofop data, a twoparameter log-logistic regression was fit using the LL.4 [drc] function:

$$
y=c+\frac{d-c}{1+\exp (m[\log (x)-\tilde{e}])}
$$

where lower limit $c$ is fixed at 0 , upper limit $d$ is fixed at $100, m$ is the slope, and $e$ is the $50 \%$ growth reducing dose $\left(\mathrm{GR}_{50}\right)$. A four-parameter Brain-Cousens model was fit using the BC.4 [drc] function for haloxyfop data:

$$
y=c+\frac{d-c+f}{1+\exp (m[\log (x)-\tilde{e}])}
$$

where lower limit $c$ is fixed at $0, d$ is the upper limit, $f$ is the hormesis effect, with $m$ and $e$ as additional parameters without direct interpretation. The $\mathrm{GR}_{50}$ of the haloxyfop model was estimated through parameterization with the $\mathrm{ED}$ [drc] function. A resistance factor $\left(\mathrm{R}_{\mathrm{S}} \mathrm{S}_{50}\right)$ was calculated by dividing $\mathrm{GR}_{50}$ 's of quizalofop-resistant wheat lines by the $\mathrm{GR}_{50}$ of a wildtype, susceptible line with the EDcomp [drc] function. Student's t-tests $(\mathrm{a}=0.05)$ of resistance factor ratios were used to identify significant fold differences in resistance between estimated $\mathrm{GR}_{50}$ doses.

A linear calibration curve of absorbance versus BSA concentration was fit using the stats [lm] function to estimate wheat ACCase extract protein concentrations ${ }^{36}$ :

$$
y=m x+b
$$

where $m$ and $b$ constants and standard deviations were $2.76 \cdot 10^{-3} \pm 3.21 \cdot 10^{-4}$ and $1.40 \cdot 10^{-1} \pm 1.32 \cdot 10^{-3}$, respectively. The residual standard error of the calibration was $1.06 \cdot 10^{-3}$ with a multiple R-squared value of 0.987 . Genotype protein concentrations were calculated from the calibration and adjusted for dilution.

Per genotype, the DPM measurement of a reaction to which water was added instead of acetyl-CoA was subtracted from replicate specific activity measurements as background activity. Mean DPM enzyme specific activity measurements were converted to ${ }^{14} \mathrm{C}$ pmol units using manufacturer provided activity of ${ }^{14} \mathrm{C}$-labeled sodium bicarbonate. Specific activity was then weighted by protein concentration to yield units of ${ }^{14} \mathrm{C} \mathrm{pmol} \mathrm{mg}^{-1}$ protein. Standard errors reflect error propagation of DPM measurement replicates and protein concentration. Specific activity was compared between genotypes using ordinary one-way ANOVA F-protected Student's t-tests $(\mathrm{a}=0.05, \mathrm{n}=3)$ with anova [stats] and emmeans [emmeans] functions.

For specific activity dose-response analysis, background activity was subtracted from DPM readings. Background corrected DPM readings were normalized to percent residual activity using control means for each genotype. Data was modelled using the same methods as for the effect of quizalofop on whole-plant growth, with the exception that $e$ represents the $50 \%$ enzyme inhibition dose $\left(\mathrm{I}_{50}\right)$. Similar resistance factors $(\mathrm{R}: \mathrm{S})$ were calculated using $\mathrm{I}_{50}$ estimates, followed by Student's t-tests $(\mathrm{a}=0.05)$ of ratios to identify significant fold differences in resistance between estimated doses.

\section{Data availability}

The datasets generated during and/or analyzed during the current study are available from the corresponding author on reasonable request.

Received: 27 August 2021; Accepted: 20 December 2021

Published online: 13 January 2022

\section{References}

1. Sasaki, Y. \& Nagano, Y. Plant acetyl-CoA carboxylase: Structure, biosynthesis, regulation, and gene manipulation for plant breeding. Biosci. Biotechnol. Biochem. 68, 1175-1184 (2004).

2. Takano, H. K., Ovejero, R. F. L., Belchior, G. G., Maymone, G. P. L. \& Dayan, F. E. ACCase-inhibiting herbicides: Mechanism of action, resistance evolution and stewardship. Scientia Agricola 78, e20190102. https://doi.org/10.1590/1678-992X-2019-0102 (2021).

3. Konishi, T., Shinohara, K., Yamada, K. \& Sasaki, Y. Acetyl-CoA carboxylase in higher plants: Most plants other than gramineae have both the prokaryotic and the eukaryotic forms of this enzyme. Plant Cell Physiol. 37, 117-122 (1996).

4. Sasaki, Y., Konishi, T. \& Nagano, Y. The compartmentation of acetyl-coenzyme A carboxylase in plants. Plant Physiol. 108, 445-449 (1995). 
5. Nikolskaya, T., Zagnitko, O., Tevzadze, G., Haselkorn, R. \& Gornicki, P. Herbicide sensitivity determinant of wheat plastid acetylCoA carboxylase is located in a 400-amino acid fragment of the carboxyltransferase domain. Proc. Natl. Acad. Sci. USA 96, 14647-14651. https://doi.org/10.1073/pnas.96.25.14647 (1999).

6. Dayan, F. E. et al. in Comprehensive Biotechnology Vol. 4 (ed Bernard Grodzinski) 36-48 (Elsevier, Amsterdam, 2020).

7. Bough, R. et al. The CoAXium wheat production system: A new herbicide-resistant system for annual grass weed control and integrated weed management. Outlooks Pest Manag. 32(4), 151-157. https://doi.org/10.1564/v32_aug_04 (2021).

8. Gornicki, P. et al. Plastid-localized acetyl-CoA carboxylase of bread wheat is encoded by a single gene on each of the three ancestral chromosome sets. Proc. Natl. Acad. Sci. USA 94, 14179-14184 (1997).

9. Gornicki, P. et al. Wheat acetyl-coenzyme A carboxylase: cDNA and protein structure. Proc. Natl. Acad. Sci. USA 91, 6860-6864 (1994).

10. Burton, J. D. et al. Kinetics of inhibition of acetyl-coenzyme A carboxylase by sethoxydim and haloxyfop. Pestic. Biochem. Physiol. 39, 100-109. https://doi.org/10.1016/0048-3575(91)90130-E (1991).

11. Rendina, A. R. et al. Kinetic characterization, stereoselectivity, and species selectivity of the inhibition of plant acetyl-CoA carboxylase by the aryloxyphenoxypropionic acid grass herbicides. Arch. Biochem. Biophys. 265, 219-225. https://doi.org/10.1016/ 0003-9861(88)90387-6 (1988).

12. Zhang, H., Tweel, B. \& Tong, L. Molecular basis for the inhibition of the carboxyltransferase domain of acetyl-coenzyme-A carboxylase by haloxyfop and diclofop. Proc. Natl. Acad. Sci. USA 101, 5910-5915. https://doi.org/10.1073/pnas.0400891101 (2004).

13. Yu, L. P. C., Kim, Y. S. \& Tong, L. Mechanism for the inhibition of the carboxyltransferase domain of acetyl-coenzyme A carboxylase by pinoxaden. Proc. Natl. Acad. Sci. USA 107, 22072-22077. https://doi.org/10.1073/pnas.1012039107 (2010).

14. Ostlie, M. et al. Development and characterization of mutant winter wheat (Triticum aestivum L.) accessions resistant to the herbicide quizalofop. Theor. Appl. Genet. 128, 343-351. https://doi.org/10.1007/s00122-014-2434-4 (2015).

15. Nandula, V. K. et al. Herbicide metabolism: Crop selectivity, bioactivation, weed resistance, and regulation. Weed Sci. 67, 149-175. https://doi.org/10.1017/wsc.2018.88 (2019).

16. Shaner, D. L. Herbicide Handbook 10th edn. (Weed Science Society of America, 2014).

17. Herbicide Resistance Action Committee (HRAC). https://hracglobal.com/.

18. Bough, R. A., Gaines, T. A. \& Dayan, F. E. Low temperature delays metabolism of quizalofop in resistant winter wheat and three annual grass weed species. Front. Agron. https://doi.org/10.3389/fagro.2021.800731 (2021).

19. Yu, Q., Friesen, L. J. S., Zhang, X.-Q. \& Powles, S. B. Tolerance to acetolactate synthase and acetyl-coenzyme A carboxylase inhibiting herbicides in Vulpia bromoides is conferred by two co-existing resistance mechanisms. Pestic. Biochem. Physiol. 78, 21-30 (2004).

20. Seefeldt, S. S. et al. Mechanisms of resistance to diclofop of two wild oat (Avena fatua) biotypes from the Willamette Valley of Oregon. Weed Sci. 44, 776-781 (1996).

21. Dayan, F. E. et al. Biochemical markers and enzyme assays for herbicide mode of action and resistance studies. Weed Sci. 63, 23-63. https://doi.org/10.1614/WS-D-13-00063.1 (2015).

22. Li, W. et al. The EMBL-EBI bioinformatics web and programmatic tools framework. Nucleic Acids Res. 43, W580-W584. https:// doi.org/10.1093/nar/gkv279 (2015).

23. Brunharo, C. A. C. G., Takano, H. K., Mallory-Smith, C. A., Dayan, F. E. \& Hanson, B. D. Role of glutamine synthetase isogenes and herbicide metabolism in the mechanism of resistance to glufosinate in Lolium perenne L. spp. multiflorum biotypes from Oregon. J. Agric. Food Chem. 67, 8431-8440. https://doi.org/10.1021/acs.jafc.9b01392 (2019).

24. Sali, A., Potterton, L., Yuan, F., Van Vlijmen, H. \& Karplus, M. Evaluation of comparative protein modeling by MODELLER. Proteins 23, 318-326. https://doi.org/10.1002/prot.340230306 (1995).

25. Webb, B. \& Sali, A. Comparative protein structure modeling using MODELLER. Curr. Protoc. Bioinf. 47, 5.6.1-5.6.32. https://doi. org/10.1002/0471250953.bi0506s47 (2014).

26. Webb, B. \& Sali, A. in Functional Genomics: Methods in Molecular Biology Vol. 1654 (eds Michael Kaufmann, Claudia Klinger, \& Andreas Savelsbergh) 39-54 (Humana Press, 2017).

27. Abraham, M. J. et al. GROMACS: High performance molecular simulations through multi-level parallelism from laptops to supercomputers. SoftwareX 1-2, 19-25. https://doi.org/10.1016/j.softx.2015.06.001 (2015).

28. Hess, B., Kutzner, C., van der Spoel, D. \& Lindahl, E. GROMACS 4: Algorithms for highly efficient, load-balanced, and scalable molecular simulation. J. Chem. Theor. Comput. 4, 435-447. https://doi.org/10.1021/ct700301q (2008).

29. Chen, V. B. et al. MolProbity: All-atom structure validation for macromolecular crystallography. Act. Crystall. D: Biol. Crystall. 66, 12-21 (2010).

30. Davis, I. W. et al. MolProbity: All-atom contacts and structure validation for proteins and nucleic acids. Nucleic Acids Res. 35, W375-W383. https://doi.org/10.1093/nar/gkm216 (2007).

31. Delano, W. L. PyMOL: An open-source molecular graphics tool. Ccp4 Newslett Protein Crystallogr. 40, 11 (2002).

32. Goodsell, D. S., Sanner, M. F., Olson, A. J. \& Forli, S. The AutoDock suite at 30. Protein Sci. 30, 31-43. https://doi.org/10.1002/pro. 3934 (2021).

33. Morris, G. M. et al. AutoDock Version 4.2. Automated Docking of Flexible Ligands to Flexible Receptors (2012).

34. Gasteiger, J. \& Marsili, M. Iterative partial equalization of orbital electronegativity: A rapid access to atomic charges. Tetrahedron 36, 3219-3222 (1980).

35. R: A language and environment for statistical computing v. 3.3.3 (R Foundation for Statistical Computing, 2015).

36. Ritz, C. \& Streibig, J. C. Bioassay analysis using R. J. Stat. Softw. 12, 1-22 (2005).

\section{Acknowledgements}

This research was funded by the Colorado Wheat Research Foundation grant number 5303094 and the USDA National Institute of Food and Agriculture, Hatch Project 1016591, COL00785.

\section{Author contributions}

R.B. and F.D. planned the experiments, carried out the experiments, generated figures and wrote the manuscript.

\section{Competing interests}

The authors declare no competing interests.

\section{Additional information}

Correspondence and requests for materials should be addressed to F.E.D.

Reprints and permissions information is available at www.nature.com/reprints.

Publisher's note Springer Nature remains neutral with regard to jurisdictional claims in published maps and institutional affiliations. 
(c) (i) Open Access This article is licensed under a Creative Commons Attribution 4.0 International cc) License, which permits use, sharing, adaptation, distribution and reproduction in any medium or format, as long as you give appropriate credit to the original author(s) and the source, provide a link to the Creative Commons licence, and indicate if changes were made. The images or other third party material in this article are included in the article's Creative Commons licence, unless indicated otherwise in a credit line to the material. If material is not included in the article's Creative Commons licence and your intended use is not permitted by statutory regulation or exceeds the permitted use, you will need to obtain permission directly from the copyright holder. To view a copy of this licence, visit http://creativecommons.org/licenses/by/4.0/.

(C) The Author(s) 2022 